\title{
Output Selection and Observer Design for Boolean Control Networks: A Sub-Optimal Polynomial-Complexity Algorithm
}

\author{
Eyal Weiss and Michael Margaliot
}

\begin{abstract}
Using a graph-theoretic approach, we derive a new sufficient condition for observability of a Boolean control network (BCN). Based on this condition, we describe two algorithms: the first selects a set of nodes so that observing this set makes the $\mathrm{BCN}$ observable. The second algorithm builds an observer for the observable $\mathrm{BCN}$. Both algorithms are suboptimal, as they are based on a sufficient but not necessary condition for observability. Yet their time-complexity is linear in the length of the description of the $\mathrm{BCN}$, rendering them feasible for large-scale networks. We discuss how these results can be used to provide a sub-optimal yet polynomial-complexity algorithm for the minimal observability problem in BCNs. Some of the theoretical results are demonstrated using a BCN model of the core network regulating the mammalian cell cycle.
\end{abstract}

\section{INTRODUCTION}

Boolean networks (BNs) are discrete-time dynamical systems with Boolean state-variables (SVs) and Boolean update functions. BNs have found wide applications in modeling and analysis of dynamical systems. They have been used to capture the existence and directions of links in complex systems (see, e.g., [30]), to model social networks (see, e.g. [14], [22]), and the spread of epidemics [20].

Recently, BNs are extensively used in systems biology (see, e.g. [17], [32]). A typical example is modeling gene regulation networks using $\mathrm{BNs}$ (see, e.g. [21], [1]). Here the state of each gene, that may be expressed or not, is modeled using a Boolean SV. The interactions between the genes (e.g. through the effect of the proteins that they encode on the promoter regions of other genes) determine the Boolean update function for each SV.

BNs with (Boolean) control inputs are called Boolean control networks (BCNs). Cheng and his colleagues [5] used the semi-tensor product (STP) of matrices to represent the dynamics of a $\mathrm{BCN}$ in a form similar to that of a discrete-time linear control system. This led to the analysis of many control-theoretic problems for $\mathrm{BCNs}$, including controllability [7], [23] observability [10], [7], disturbance decoupling [6], stabilization by state feedback [26] and optimal control [11], [35], [25]. However, the STP representation is exponential in the number of Boolean SVs, and thus it cannot be used in general to develop efficient algorithms for addressing these problems.

In many real-world systems it is not possible to measure the state of all the SVs. For instance, the function of a

This research is supported in part by a research grant from the Israel Science Foundation (ISF grant 410/15).

The authors are with the School of Elec. Eng., Tel-Aviv University, Israel 69978. Corresponding Author: michaelm@eng.tau.ac.il multipolar neuron may depend upon signals received from thousands of other interconnected neurons (see, e.g., [15]).

State observers are needed to reconstruct the entire state of the system based on a time sequence of what can be measured, i.e. the system outputs. An estimate of the entire state is useful in many applications, as it greatly assists in monitoring and control. For example, in bio-reactors one may use sensors to measure variables such as dissolved oxygen, $\mathrm{pH}$ and temperature, yet key biological state-variables such as biomass and product concentrations can be much more difficult (and costly) to measure [16]. A typical application of state-observers is to integrate their estimates in full-state feedback controllers (see, e.g., [31]).

The existence of observers is known to depend on the reconstructability property of the system, which was shown to be equivalent to another property, called observability, in linear systems [19] and in BNs [10]. In BCNs, there are several different definitions for observability appearing in the literature [7], [37], [24], [10] (see also the work on final state observable graphs in [18]), making the connection to reconstructability somewhat complex. In subsection I-B we state the definition used in this paper and clarify its relation to the definition of reconstructability.

When a given system is not observable, it is sometimes possible to make it observable by placing additional sensors that measure more (functions of the) SVs. Of course, this may be costly in terms of resources, so a natural question is: find the minimal number of measurements to add so that the resulting system is observable. This minimal observability problem is also interesting theoretically, as its solution means identifying the (functions of) SVs that provide the maximal information on the entire state of the system [27]. Indeed, minimal observability problems have recently attracted considerable interest. Examples include monitoring complex services by minimal logging [2], the optimal placement of phasor measurement units in power systems (see, e.g., [28]), and the minimal sparse observability problem addressed in [29].

It is known that testing observability of BCNs is NP-hard in the number of SVs of the system [24] (for a general survey on the computational complexity of various problems in systems and control theory, see [3]). This means that, unless $\mathrm{P}=\mathrm{NP}$, it is computationally intractable to determine whether a large $\mathrm{BCN}$ is observable. This implies that the minimal observability problem in BCNs is also NP-hard, since it must entail analyzing observability.

It is thus not surprising that many observers for BCNs have exponential complexity. These include the Shift-Register ob- 
server, the Multiple States observer [10] and the Luenbergerlike observer [36]. This complexity implies that these algorithms cannot be used in large-scale networks.

This paper is motivated by recent work on the minimal observability problem in a special class of BNs called conjunctive Boolean networks (CBNs). In a $\mathrm{CBN}$ every update function is comprised of only AND operations. A necessary and sufficient observability condition for CBNs has been derived in [33]. This condition is based on a graph representing the dependencies between the SVs and the update functions. This yields polynomial-time algorithms for: (1) solving the minimal observability problem in CBNs; and (2) designing observers for observable CBNs.

Here, we show that a similar graph-theoretic approach provides a sufficient (but not necessary) condition for observability of (general) BCNs. This induces algorithms for solving Problems (1) and (2) above. Now the algorithms are not optimal anymore, i.e. they may add more observations than the minimal number that is indeed required. Nevertheless, they retain their polynomial-time complexity implying that they are feasible for large-scale BCNs. Of course, in the particular case where all the update functions are AND gates these algorithms become optimal.

We note in passing that the special structure of CBNs makes them amenable to analysis (see, e.g. [13], [4], [12], [34]) and we believe that more results from this field can be extended to handle general BNs.

The next section reviews some known definitions and results that are used in deriving the main results in Section III

\section{Preliminaries}

\section{A. Boolean Control Networks}

Let $S:=\{0,1\}$. For two integers $i, j$ let $[i, j]:=\{i, i+$ $1, \ldots, j\}$. A BCN with $n \mathrm{SVs}, p$ inputs and $m$ outputs can be represented by the following equations:

$$
\begin{array}{rlrl}
X_{i}(k+1) & =f_{i}(X(k), U(k)), & & \forall i \in[1, n], \\
Y_{j}(k) & =h_{j}(X(k)), & \forall j \in[1, m],
\end{array}
$$

where the state vector at time $k$ is denoted by $X(k):=$ $\left[\begin{array}{lll}X_{1}(k) & \ldots & X_{n}(k)\end{array}\right]^{\prime} \in S^{n}$, the input vector by $U(k):=$ $\left[\begin{array}{lll}U_{1}(k) & \ldots & U_{p}(k)\end{array}\right]^{\dagger} \in S^{p}$, the output vector by $Y(k):=$ $\left[\begin{array}{lll}Y_{1}(k) & \ldots & Y_{m}(k)\end{array}\right]^{\prime} \in S^{m}$ and $f_{i}, h_{j}$ are Boolean functions.

In principle, an update function may include an SV (or control input) that has no effect on the function, e.g. $f_{1}\left(X_{1}(k), X_{2}(k)\right)=X_{1}(k) \vee\left(X_{2}(k) \wedge \bar{X}_{2}(k)\right)$. We assume that such arguments have been removed, in other words, if $X_{i}(k)$ is an argument of $f_{j}$ then there exists an assignment of $X_{1}, \ldots, X_{i-1}, X_{i+1}, \ldots, X_{n}, U$ such that

$$
\begin{aligned}
& f_{j}\left(X_{1}, \ldots, X_{i-1}, 0, X_{i+1}, \ldots, X_{n}, U\right) \\
\neq & f_{j}\left(X_{1}, \ldots, X_{i-1}, 1, X_{i+1}, \ldots, X_{n}, U\right) .
\end{aligned}
$$

Similarly, a control input appears in an update function only if it really affects the function.
If for some $i$ there exists an output $Y_{j}(k)=X_{i}(k)$ then we say that the SV $X_{i}$ is directly observable or directly measurable.

\section{B. Observability and Reconstructability}

Observability [reconstructability] refers to the ability to uniquely determine the initial [final] state of a system based on a time-sequence of the input and output. There are several definitions in the literature for observability of BCNs. Here, we follow the one used in [10].

Definition 1. We say that (1) is observable on $[0, N]$ if for every control sequence $U:=\{U(0), \ldots, U(N-1)\}$ and for any two different initial conditions $X(0)$ and $\tilde{X}(0)$ the corresponding solutions of the BCN for $U$ yield different output sequences $\{Y(0), \ldots, Y(N)\}$ and $\{\tilde{Y}(0), \ldots, \tilde{Y}(N)\}$.

This means that it is always possible to uniquely determine the initial condition from the output sequence on $[0, N]$. A BCN is called observable if it is observable for some value $N \geq 0$.

Definition 2. [10] The BCN (1) is said to be reconstructable if for any integer $r>0$ the knowledge of every admissible input and output trajectories $\{(Y(k), U(k))\}, k=0,1, \ldots, r$, uniquely determine the final state $X(r)$.

Clearly, if a $\mathrm{BCN}$ is observable then it is also reconstructable.

\section{Directed Graphs}

Let $G=(V, E)$ be a directed graph (digraph), with $V$ the set of vertices, and $E$ the set of directed edges (arcs). Let $e_{i \rightarrow j}$ (or $\left(v_{i} \rightarrow v_{j}\right)$ ) denote the arc from $v_{i}$ to $v_{j}$. When such an arc exists, we say that $v_{i}$ is an in-neighbor of $v_{j}$, and $v_{j}$ as an out-neighbor of $v_{i}$. The set of in-neighbors [outneighbors] of $v_{i}$ is denoted by $\mathcal{N}_{\text {in }}\left(v_{i}\right)\left[\mathcal{N}_{\text {out }}\left(v_{i}\right)\right]$. The indegree [out-degree] of $v_{i}$ is $\left|\mathcal{N}_{\text {in }}\left(v_{i}\right)\right|\left[\left|\mathcal{N}_{\text {out }}\left(v_{i}\right)\right|\right]$. A source [sink] is a node with in-degree [out-degree] zero.

For $v_{i}, v_{j} \in V$ a walk from $v_{i}$ to $v_{j}$, denoted $w_{i j}$, is a sequence: $v_{i_{0}} v_{i_{1}} \ldots v_{i_{q}}$, with $v_{i_{0}}=v_{i}, v_{i_{q}}=v_{j}$, and $e_{i_{k} \rightarrow i_{k+1}} \in E$ for all $k \in[0, q-1]$. A closed walk is a walk that starts and terminates at the same vertex. A closed walk is called a cycle if all the vertices in the walk are distinct, except for the start-vertex and the end-vertex.

\section{Dependency Graph}

The dependency graph of a $\mathrm{BCN}$ is defined by $G=$ $(V, E)$, where $V=\left\{X_{1}, \ldots, X_{n}, U_{1}, \ldots, U_{p}\right\}$ i.e. every vertex corresponds to either an SV or an input of the BCN. The edge $\left(X_{i} \rightarrow X_{j}\right) \in E\left[\left(U_{q} \rightarrow X_{j}\right) \in E\right]$ iff $X_{i}(k)\left[U_{q}(k)\right]$ is an argument of $f_{j}$ (the update function of $\left.X_{j}(k+1)\right)$. Thus, the dependency graph encodes the actual variable dependencies in the update functions.

We denote the subset of nodes corresponding to SVs by $V_{s}:=\left\{X_{1}, \ldots, X_{n}\right\} \subseteq V$, the subset of edges involving only SVs by $E_{s} \subseteq E$, and let $G_{s}:=\left(V_{s}, E_{s}\right)$ be the resulting "reduced" dependency graph. Referring to the outputs of the $\mathrm{BCN}$, we say that a node in the dependency graph that 
represents a [non] directly observable SV is a [non] directly observable node.

\section{MAin Results}

From here on, we consider BCNs in the form:

$$
\begin{array}{rlrl}
X_{i}(k+1) & =f_{i}(X(k), U(k)), & & i \in\{1, \ldots, n\}, \\
Y_{j}(k) & =X_{j}(k), & j \in\{1, \ldots, m\},
\end{array}
$$

that is, every output $Y_{j}$ is the value of an SV. We assume without loss of generality that the $m$ outputs correspond to the first $m$ SVs. Thus, nodes $X_{1}, \ldots, X_{m}\left[X_{m+1}, \ldots, X_{n}\right]$ in the dependency graph are [non] directly observable.

\section{A. Sufficient Condition for Observability}

We begin by presenting two definitions which will be used later on.

Definition 3. We say that a $B C N$ has Property $P_{1}$ if for every non-directly observable node $X_{i}$ there exists some other node $X_{j}$ such that $\mathcal{N}_{i n}\left(X_{j}\right)=\left\{X_{i}\right\}$.

In this case, $X_{j}(k+1)=f_{j}\left(X_{i}(k)\right)$, and thus either $X_{j}(k+1)=X_{i}(k)$ or $X_{j}(k+1)=\bar{X}_{i}(k)$. This means that the information on the state of $X_{i}$ "propagates" to $X_{j}$.

Definition 4. We say that a $B C N$ has Property $P_{2}$ if every cycle $C$ in its dependency graph that is composed solely of nondirectly observable nodes satisfies the following property: $C$ includes a node $X_{i}$ which is the only element in the inneighbors set of some other node $X_{j}$, i.e. $N_{i n}\left(X_{j}\right)=\left\{X_{i}\right\}$, and $X_{j}$ is not part of the cycle $C$.

This means that $X_{j}(k+1)=f_{j}\left(X_{i}(k)\right)$, so the information on the state of every node in the cycle propagates to $X_{i}$ and then to $X_{j}$, where $X_{j}$ is not part of the cycle.

We now provide a sufficient condition for observability.

Theorem 1. A BCN that satisfies properties $P_{1}$ and $P_{2}$ is observable.

To prove this, we first introduce another definition and several auxiliary results.

Definition 5. An observed path in the dependency graph is a non-empty ordered set of nodes such that: (1) the last element in the set is a directly observable node; and (2) if the set contains $p>1$ elements, then for any $i<p$ the $i$-th element is a non-directly observable node, and is the only element in the in-neighbors set of node $i+1$. Observed paths with non-overlapping nodes are called disjoint observed paths.

Roughly speaking, an observed path corresponds to a "shift register" whose last cell is directly observable.

Proposition 1. Consider a BCN that satisfies properties $P_{1}$ and $P_{2}$. Then:

1) $G_{s}$ can be decomposed into disjoint observed paths, such that every vertex in $G_{s}$ belongs to a single observed path (i.e., the union of the disjoint observed paths is a vertex cover of $G_{s}$ ).
2) For every vertex $v \in\left(V \backslash V_{s}\right), \mathcal{N}_{\text {out }}(v)$ contains only vertices that are located at the beginning of observed paths.

Proof of Prop. 1. We give a constructive proof. Algorithm 1 below accepts a graph $G$ that satisfies properties $P_{1}$ and $P_{2}$ and terminates after each vertex in $G_{s}$ belongs to exactly one observed path. Comments in the algorithm are enclosed within $(* \ldots *)$.

Algorithm 1 Decompose the nodes of $G_{s}$ into disjoint observed paths

Input: Dependency graph $G$ of a BCN in the form (2) that satisfies properties $P_{1}$ and $P_{2}$.

Output: A decomposition of $G_{s}$ into $m$ disjoint observed paths.

1: for $i=1$ to $m$ do (* every iteration builds a new path ending with $X_{i} *$ )

2: $\quad$ o-node $\leftarrow X_{i} ;$ o-path $\leftarrow\left\{X_{i}\right\}$

3: $\quad$ if $\mid \mathcal{N}_{\text {in }}($ o-node $) \mid=1$ then

4: $\quad$ Let $v$ be such that $\{v\}=\mathcal{N}_{\text {in }}$ (o-node)

5: $\quad$ if $v$ does not belong to a previous path, $v \in V_{s}$

6: $\quad$ and $v$ is not directly observable then

7: $\quad$ insert $v$ to o-path just before $o$-node

8: $\quad$ o-node $\leftarrow v$; goto 3

9: $\quad$ else print $o$-path

10: end for

We now prove the correctness of Algorithm 1. To simplify the notation, we say that $v_{p}$ points to $v_{q}$ if $p \neq q$ and $N_{i n}\left(v_{q}\right)=\left\{v_{p}\right\}$, and denote this by $v_{p} \mapsto v_{q}$. The special arrow indicates that the dependency graph includes an edge from $v_{p}$ to $v_{q}$ and that there are no other edges pointing to $v_{q}$.

If all the nodes of $G_{s}$ are directly observable (i.e. if $m=$ $n$ ) the algorithm will assign every node to a different observed path and this is correct. Thus, we may assume that $m<n$. Pick a non directly observable node $X_{j}$. Then $m<j \leq n$. Our first goal is to prove the following result.

Claim 1. The algorithm outputs an observed path that contains $X_{j}$.

By Property $P_{1}$, there exists $k \neq j$ such that $X_{j} \mapsto X_{k}$. We consider two cases.

Case 1. If $k \leq m$ then $X_{k}$ is directly observable and the algorithm will add $X_{j}$ to an observed path as it "traces back" from $X_{k}$ unless $X_{j}$ has already been included in some other observed path found by the algorithm. Thus, in this case Claim 1 holds.

Case 2. Suppose that $k>m$, i.e. $X_{k}$ is non directly observable. By Property $P_{1}$, there exists $h \neq k$ such that $X_{k} \mapsto X_{h}$, so $X_{j} \mapsto X_{k} \mapsto X_{h}$. If $h \leq m$ then we conclude as in Case 1 that the algorithm outputs an observed path that contains $X_{j}$. Thus, we only need to consider the case where as we proceed from $X_{j}$ using Property $P_{1}$ we never "find" a directly observable node. Then there exists a set of non 
directly observable nodes $X_{k_{1}}, \ldots, X_{k_{\ell}}$, with $k_{1}=j$, such that $X_{k_{1}} \mapsto X_{k_{2}} \mapsto \cdots \mapsto X_{k_{\ell}} \mapsto X_{k_{1}}$. This means that $X_{j}$ is part of a cycle $C$ of non directly observable nodes. By Property $P_{2}, C$ includes a node $X_{k_{i}}$ such that $X_{k_{i}} \mapsto X_{s_{1}}$, where $X_{s_{1}}$ is not part of the cycle $C$. If $X_{s_{1}}$ is directly observable then we conclude that the algorithm will output an observed path that includes $X_{j}$. If $X_{s_{1}}$ is not directly observable then by Property $P_{1}$, there exists $s_{2} \neq s_{1}$ such that $X_{s_{1}} \mapsto X_{s_{2}}$. Furthermore, since every node in $C$ has in degree one, $X_{s_{2}} \notin C$. Proceeding this way, we conclude that there exist $s_{1}, \ldots, s_{p}$ such that $X_{k_{i}} \mapsto X_{s_{1}} \mapsto X_{s_{2}} \mapsto \cdots \mapsto$ $X_{s_{p}}$, with $X_{s_{p}}$ a directly observable node. This means that the algorithm will output $X_{j}$ in an observed path as it traces back from $X_{s_{p}}$, unless it already included $X_{j}$ in another observed path. This completes the proof of Claim 1

Summarizing, we showed that every non directly observable node $X_{j}$ is contained in an observed path produced by the algorithm. The fact that every $X_{j}$ will be in a single observed path, and that the observed paths will be distinct is clear from the description of the algorithm. From the definition of an observed path, it is clear that only a vertex which is located at the beginning of an observed path may contain edges from vertices in $V \backslash V_{s}$. This completes the proof of Prop. 1.

We can now prove Thm. 1

Proof of Thm. 1. Consider the following three statements:

(a) The dependency graph $G$ has Properties $P_{1}$ and $P_{2}$;

(b) There exists a decomposition of the dependency graph into $m \geq 1$ disjoint observed paths $O^{1}, \ldots, O^{m}$, such that every vertex in $G$ belongs to a single observed path.

(c) The BCN is observable.

The correctness of Algorithm 1 implies that $(a) \rightarrow(b)$. We now show that $(b) \rightarrow(c)$. Suppose that $(b)$ holds. Let $O^{i}=$ $\left(X_{i_{1}}, \ldots, X_{i_{N_{i}}}\right)$ be an observed path. The output of this path at times $0, \ldots, N_{i}-1$, is

$$
\begin{aligned}
X_{i_{N_{i}}}(0) & =X_{i_{N_{i}}}(0), \\
X_{i_{N_{i}}}(1) & =f_{i_{N_{i}}}\left(X_{i_{N_{i}-1}}(0)\right), \\
X_{i_{N_{i}}}(2) & =f_{i_{N_{i}}}\left(f_{i_{N_{i}-1}}\left(X_{i_{N_{i}-2}}(0)\right)\right), \\
& \vdots \\
X_{i_{N_{i}}}\left(N_{i}-1\right) & =f_{i_{N_{i}}}\left(f_{i_{N_{i}-1}}\left(\ldots f_{i_{2}}\left(X_{i_{1}}(0)\right)\right)\right) .
\end{aligned}
$$

Each of $f_{i_{1}}, \ldots, f_{i_{N_{i}}}$ is either the identity function or the NOT operator, so they are all invertible. Moreover, their inverse functions are the functions themselves, namely, $f_{i_{1}}^{-1}=f_{i_{1}}, \ldots, f_{i_{N_{i}}}^{-1}=f_{i_{N_{i}}}$. Hence, the initial values of the SVs in $O^{i}$ can be reconstructed as $X_{i_{N_{i}}}(0), X_{i_{N_{i}-1}}(0)=$ $f_{i_{N_{i}}}\left(X_{i_{N_{i}}}(1)\right), X_{i_{N_{i}-2}}(0)=f_{i_{N_{i}-1}}\left(f_{i_{N_{i}}}\left(X_{i_{N_{i}}}(2)\right)\right)$, and so on. We conclude that it is possible to determine the initial condition of every SV in the BCN using the output sequence on $\left[0, \max _{i=1, \ldots, m}\left\{N_{i}\right\}-1\right]$, and this holds for every control sequence. Thus, the $\mathrm{BCN}$ is observable, so $(b) \rightarrow(c)$. We conclude that $(a) \rightarrow(c)$ and this proves Thm. 1

\section{B. Observer Design}

The proof of Thm. 1 implies the following result.

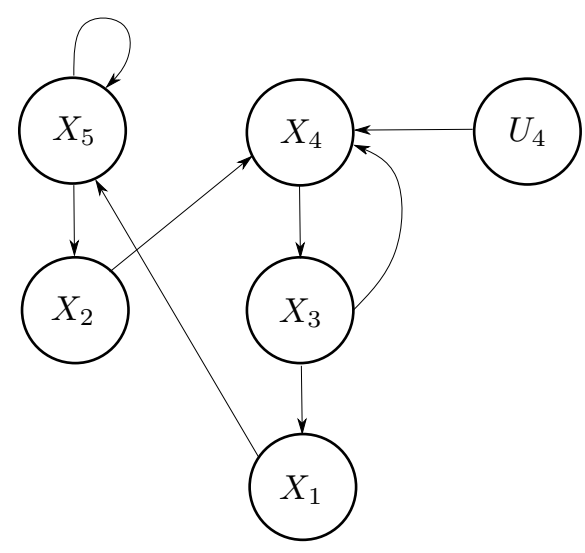

Fig. 1. The dependency graph $G$ of the BCN in Example 1

Corollary 1. Consider a $B C N$ that satisfies the sufficient condition stated in Thm. 1 An observer for this BCN can be designed as follows:

(a) Construct the dependency graph $G$;

(b) Apply Algorithm 1 to decompose the nodes of $G_{s}$ into a set of disjoint observed paths;

(c) Observe an output sequence of length equal to the longest observed path;

(d) Map the values observed at each output to the values of the SVs composing the observed paths, as done in the proof of Thm. प to obtain the initial state $X(0)$.

Of course, once the initial condition is determined the state for all time $k$ can be obtained using the known input sequence and dynamics. We refer to the observer described above as the Disjoint-Path Observer.

Example 1. Consider the single-input and two-output BCN:

$$
\begin{aligned}
X_{1}(k+1) & =X_{3}(k), \\
X_{2}(k+1) & =\bar{X}_{5}(k), \\
X_{3}(k+1) & =\bar{X}_{4}(k), \\
X_{4}(k+1) & =\left(X_{2}(k) \wedge X_{3}(k)\right) \vee U_{4}(k), \\
X_{5}(k+1) & =\bar{X}_{1}(k) \vee X_{5}(k), \\
Y_{1}(k) & =X_{1}(k), \\
Y_{2}(k) & =X_{2}(k) .
\end{aligned}
$$

The dependency graph $G$ of this BCN is depicted Fig. $\square$ and it is straightforward to verify that it satisfies Properties $P_{1}, P_{2}$. Thm. $\square$ implies that this $B C N$ is observable, and that $G_{s}$ is decomposable to a set of disjoint observed paths. Applying Algorithm 1 to this BCN yields a decomposition to two observed paths: $O^{1}=\left(X_{4}, X_{3}, X_{1}\right), O^{2}=\left(X_{5}, X_{2}\right)$, where $X_{4} \mapsto X_{3} \mapsto X_{1}, X_{5} \mapsto X_{2}$.

Complexity Analysis of the Construction of a Disjoint-Path Observer: The complexity of generating the dependency graph $G$ is linear in the description of the graph, which is $O(|V|+|E|)$. The resulting graph satisfies $|V|=n+p$, $|E| \leq n^{2}+p n$, with $n$ being the number of SVs and $p$ the number of control inputs. By using a chained-list data structure for representing the dependency graph, Algorithm 1 
can be implemented in $O(n)$ time. Indeed Algorithm 1 systematically passes through each vertex of $V_{s}$ once (with $\left|V_{s}\right|=n$ ) and performs $O(1)$ operations on each such vertex. After the decomposition to disjoint observed paths takes place, determining the initial condition of the $\mathrm{BCN}$ is attained in complexity which is at most linear in the length of the description of the BCN. From this stage, determination of the state at following time steps is obtained by direct calculation of the dynamics, which is again linear in length of the description of the BCN for every time step.

Summarizing, the complexity of the construction is linear in the length of the description of the BCN, namely $O(|V|+$ $|E|)$. Thus, it satisfies the bound $O\left(n^{2}+p n\right)$.

\section{Output Selection and an Upper Bound for Minimal Observability}

In some cases, it is possible to add sensors to measure more SVs. We consider the following problem.

Problem 1. Given a $B C N$ with $n S V s$ determine a minimal set of indices $\mathcal{I} \subseteq[1, n]$, such that making each $X_{i}(k), i \in \mathcal{I}$, directly measurable yields an observable BCN.

As mentioned in Section \, this minimal observability problem in BCNs is NP-hard. We use the conditions in Thm. 1 to provide a sub-optimal yet nontrivial solution to Problem 1 . This is described by Algorithm 2 which is discussed below. Furthermore, due to Corollary 1 an efficient (polynomial-time) observer can also be obtained for a $\mathrm{BCN}$ with outputs chosen according to this scheme.

Algorithm 2 below is based on an algorithm given in [33] that solves the minimal observability problem in a special case of BCNs. In our case (general BCNs) it does not offer a minimal solution to Problem 1, but rather it solely gives a solution which satisfies the conditions in Thm. 1.

The idea behind the algorithm is simple: it first creates three lists. A list $L_{1}$ of all SVs that are not directly observable and are not the only element in the in-neighbors' set of another node; a list $L_{2}$ of all SVs that are not directly observable and are the only element in the in-neighbors set of another node; and a list $L_{C}$ of cycles composed solely out of nodes in $L_{2}$. For each cycle $C \in L_{C}$, it then checks if one of its elements appears as the only element in the inneighbors set of another node that is not part of $C$. If so, it removes $C$ from $L_{C}$. Finally, it returns the SVs in $L_{1}$ and one element from each cycle $C \in L_{C}$. Making these SVs directly observable, by adding them as outputs, yields an observable BCN.

Since the steps of the algorithm are basically described in [33], yet here it is used for general BCNs, we describe only the functional change that is depicted in the headline (i.e., input-output description).
Algorithm 2 Output selection for meeting the condition of Thm. 1] a high-level description

Input: A BCN (2) with $n \mathrm{SVs}$ and $m \geq 0$ outputs.

Output: A set of SVs so that making these SVs directly observable yields a $\mathrm{BCN}$ that satisfies conditions $P_{1}$ and $P_{2}$.

The complexity analysis of Algorithm 2 is done in [33], yielding a runtime which is linear in the length of the description of the $\mathrm{BCN}$, namely $O(|V|+|E|)$. We note that the algorithm provides a specific solution which meets the conditions of Thm. 1, but it is straightforward to modify this so that the algorithm will return the information needed to build various possible solutions which meet the conditions of theorem (this is explained in detail in [33]). If the algorithm returns an output list that is empty then the $\mathrm{BCN}$ is observable, so it can also be used to determine if a given BCN satisfies the sufficient condition for observability.

Theorem 2. Algorithm 2 provides a solution that satisfies the conditions of Thm. 1 ,

The proof of Thm. 2] is the same one that appears in [33] (but this time it has a different meaning, namely, here we consider the general class of BCNs), so we omit it. The correctness of Algorithm 2 implies the following:

Corollary 2. An upper bound for the minimal size of the solution to Problem 1 is given by the size of the solution generated by Algorithm 2.

Note that for the particular case of CBNs the upper bound provided by size of the solution generated by Algorithm 2 is tight.

\section{A Biological Example}

Faure et al. [8], [9] derived a BCN model for the mammalian cell cycle that includes nine SVs representing the activity/inactivity of nine different proteins and a single input corresponding to the activation/inactivation of a regulating protein in the cell. The BCN dynamics is given by:

$$
\begin{aligned}
x_{1}(t+1)= & \left(\bar{u}(t) \wedge \bar{x}_{3}(t) \wedge \bar{x}_{4}(t) \wedge \bar{x}_{9}(t)\right) \\
& \vee\left(x_{5}(t) \wedge \bar{u}(t) \wedge \bar{x}_{9}(t)\right) \\
x_{2}(t+1)= & \left(\bar{x}_{1}(t) \wedge \bar{x}_{4}(t) \wedge \bar{x}_{9}(t)\right) \vee\left(x_{5}(t) \wedge \bar{x}_{1}(t) \wedge \bar{x}_{9}(t)\right), \\
x_{3}(t+1)= & x_{2}(t) \wedge \bar{x}_{1}(t), \\
x_{4}(t+1)= & \left(x_{2}(t) \wedge \bar{x}_{1}(t) \wedge \bar{x}_{6}(t) \wedge\left(\overline{x_{7}(t) \wedge x_{8}(t)}\right)\right) \\
& \vee\left(x_{4}(t) \wedge \bar{x}_{1}(t) \wedge \bar{x}_{6}(t) \wedge\left(\overline{x_{7}(t) \wedge x_{8}(t)}\right)\right) \\
x_{5}(t+1)= & \left(\bar{u}(t) \wedge \bar{x}_{3}(t) \wedge \bar{x}_{4}(t) \wedge \bar{x}_{9}(t)\right) \\
& \vee\left(x_{5}(t) \wedge\left(\overline{x_{3}(t) \wedge x_{4}(t)}\right) \wedge \bar{u}(t) \wedge \bar{x}_{9}(t)\right), \\
x_{6}(t+1)= & x_{9}(t), \\
x_{7}(t+1)= & \left(\bar{x}_{4}(t) \wedge \bar{x}_{9}(t)\right) \vee x_{6}(t) \vee\left(x_{5}(t) \wedge \bar{x}_{9}(t)\right), \\
x_{8}(t+1)= & \bar{x}_{7}(t) \vee\left(x_{7}(t) \wedge x_{8}(t) \wedge\left(x_{6}(t) \vee x_{4}(t) \vee x_{9}(t)\right)\right), \\
x_{9}(t+1)= & \bar{x}_{6}(t) \wedge \bar{x}_{7}(t) .
\end{aligned}
$$

The input is considered constant in [8], that is, either $u(t) \equiv$ True or $u(t) \equiv$ False. Under this assumption, 
the BCN becomes two BNs. The simulations in [8] show that when $u(t) \equiv$ True the corresponding BN admits a globally attracting periodic trajectory composed of 7 states. The sequence of state transitions along this trajectory qualitatively matches cell cycle progression. For $u(t) \equiv$ False the BN admits a single state that is globally attracting. This state corresponds to the G0 phase (cell quiescence).

Ref. [24] used the STP representation combined with a trial and error approach to conclude that in the particular case where $u(t) \equiv$ TRUE the solution to the minimal observability problem is to make $8 \mathrm{SV}$ directly measurable, namely, $X_{1}, \ldots, X_{8}$. Note that using the STP is applicable here, as there are only nine SVs yet the transition matrix is already $2^{9} \times 2^{9}$.

Applying Algorithm 2 to the $\mathrm{BCN}$ (4) (without assuming a necessarily constant control) generates the lists: $L_{1}=$ $\left\{X_{1}, \ldots, X_{8}\right\}, L_{2}=\left\{X_{9}\right\}$, and $L_{C}=\emptyset$, and then returns $L_{1}$. Thus, in this particular case Algorithm 2 provides an optimal solution. We emphasize again that other algorithms, that require exponential run-time, are not applicable for BCNs with, say, $n \geq 30 \mathrm{SVs}$.

\section{REFERENCES}

[1] R. Albert and H. G. Othmer, "The topology of the regulatory interactions predicts the expression pattern of the segment polarity genes in Drosophila melanogaster," J. Theoretical Biology, vol. 223, pp. 1-18, 2003.

[2] D. Biswas and B. Genest, "Minimal observability for transactional hierarchical services," in Proc. 20th Int. Conf. Software Engineering and Knowledge Engineering (SEKE'2008), San Francisco, CA, 2008, pp. 531-536.

[3] V. D. Blondel and J. N. Tsitsiklis, "A survey of computational complexity results in systems and control," Automatica, vol. 36, pp. 1249-1274, 2000.

[4] X. Chen, Z. Gao, and T. Basar, "Asymptotic behavior of conjunctive Boolean networks over weakly connected digraphs," 2017. [Online]. Available: https://arxiv.org/abs/1708.01975

[5] D. Cheng, "Semi-tensor product of matrices and its application to Morgen's problem," Science in China Series: Information Sciences, vol. 44, no. 3, pp. 195-212, 2001.

[6] D. Cheng, "Disturbance decoupling of Boolean control networks," IEEE Trans. Automat. Control, vol. 56, no. 1, pp. 2-10, 2011.

[7] D. Cheng and H. Qi, "Controllability and observability of Boolean control networks," Automatica, vol. 45, no. 7, pp. 1659-1667, 2009.

[8] A. Faure, A. Naldi, C. Chaouiya, and D. Thieffry, "Dynamical analysis of a generic Boolean model for the control of the mammalian cell cycle," Bioinformatics, vol. 22, pp. e124-e131, 2006.

[9] A. Faure and D. Thieffry, "Logical modelling of cell cycle control in eukaryotes: a comparative study," Mol. BioSyst., vol. 5, pp. 15691581, 2009.

[10] E. Fornasini and M. E. Valcher, "Observability, reconstructibility and state observers of Boolean control networks," IEEE Trans. Automat. Control, vol. 58, no. 6, pp. 1390-1401, 2013.

[11] E. Fornasini and M. E. Valcher, "Optimal control of Boolean control networks," IEEE Trans. Automat. Control, vol. 59, no. 5, pp. 12581270, 2014.

[12] Z. Gao, X. Chen, and T. Basar, "Controllability of conjunctive Boolean networks with application to gene regulation," IEEE Trans. Control of Network Systems, 2018, To appear.

[13] Z. Gao, X. Chen, and T. Basar, "Stability structures of conjunctive Boolean networks," Automatica, vol. 89, pp. 8-20, 2018.

[14] D. G. Green, T. G. Leishman, and S. Sadedin, "The emergence of social consensus in Boolean networks," in Proc. 2007 IEEE Symposium on Artificial Life (IEEE-ALife'07), Honolulu, Hawaii, 2007, pp. 402-408.
[15] J. Hawkins and S. Ahmad, "Why neurons have thousands of synapses, a theory of sequence memory in neocortex," Frontiers in Neural Circuits, vol. 10, p. 23, 2016. [Online]. Available: http://journal.frontiersin.org/article/10.3389/fncir.2016.00023

[16] X. Hulhoven, A. V. Wouwer, and P. Bogaerts, "Hybrid extended Luenberger-asymptotic observer for bioprocess state estimation," Chemical Engineering Science, vol. 61, no. 21, pp. 7151-7160, 2006.

[17] I. Irurzun-Arana, J. M. Pastor, I. F. Trocniz, and J. D. GmezMantilla, "Advanced Boolean modeling of biological networks applied to systems pharmacology," Bioinformatics, vol. 33, no. 7, pp. 1040$1048,2017$.

[18] R. M. Jungers and V. D. Blondel, "Observable graphs," Discrete Applied Mathematics, vol. 159, pp. 981-989, 2011.

[19] R. E. Kalman, P. L. Falb, and M. A. Arbib, Topics in Mathematical System Theory. McGraw-Hill New York, 1969, vol. 1.

[20] A. Kasyanov, L. Kirkland, and M. T. Mihaela, "A spatial SIRS Boolean network model for the spread of H5N1 avian influenza virus among poultry farms," in Proc. 5th Int. Workshop Computational Systems Biology (WCSB), 2008, pp. 73-76.

[21] S. A. Kauffman, "Metabolic stability and epigenesis in randomly constructed genetic nets," J. Theoretical Biology, vol. 22, no. 3, pp. 437-467, 1969.

[22] S. Kochemazov and A. Semenov, "Using synchronous Boolean networks to model several phenomena of collective behavior," PLOS ONE, vol. 9, no. 12, p. e115156, 2014.

[23] D. Laschov and M. Margaliot, "Controllability of Boolean control networks via the Perron-Frobenius theory," Automatica, vol. 48, pp. 1218-1223, 2012.

[24] D. Laschov, M. Margaliot, and G. Even, "Observability of Boolean networks-a graph-theoretic approach," Automatica, vol. 49, pp. 23512362,2013

[25] D. Laschov and M. Margaliot, "A maximum principle for single-input Boolean control networks," IEEE Trans. Automat. Control, vol. 56, no. 4, pp. 913-917, 2011.

[26] R. Li, M. Yang, and T. Chu, "State feedback stabilization for Boolean control networks," IEEE Trans. Automat. Control, vol. 58, no. 7, pp. 1853-1857, 2013.

[27] Y.-Y. Liu, J.-J. Slotine, and A.-L. Barabsi, "Observability of complex systems," Proceedings of the National Academy of Sciences, vol. 110 no. 7, pp. 2460-2465, 2013.

[28] J. Peng, Y. Sun, and H. F. Wang, "Optimal PMU placement for full network observability using Tabu search algorithm," Int. J. Electrical Power \& Energy Systems, vol. 28, no. 4, pp. 223-231, 2006.

[29] S. Sarma and N. Dutt, "Minimal sparse observability of complex networks: Application to MPSoC sensor placement and run-time thermal estimation and tracking," in 2014 Design, Automation Test in Europe Conf. Exhibition (DATE'2014), 2014, pp. 1-6.

[30] S. Shahrampour and V. M. Preciado, "Topology identification of directed dynamical networks via power spectral analysis," IEEE Trans. Automat. Control, vol. 60, no. 8, pp. 2260-2265, 2015.

[31] E. D. Sontag, Mathematical Control Theory: Deterministic Finite Dimensional Systems, 2nd ed. New York: Springer, 1998.

[32] R.-S. Wang, A. Saadatpour, and R. Albert, "Boolean modeling in systems biology: an overview of methodology and applications," Physical Biology, vol. 9, no. 5, p. 055001, 2012.

[33] E. Weiss and M. Margaliot, "A polynomial-time algorithm for solving the minimal observability problem in conjunctive Boolean networks," arXiv preprint arXiv:1706.04072 2017.

[34] E. Weiss, M. Margaliot, and G. Even, "Minimal controllability of conjunctive Boolean networks is NP-complete," Automatica, vol. 92, pp. 56-62, 2018.

[35] Z. Zhang, T. Leifeld, and P. Zhang, "Finite horizon tracking control of Boolean control networks," IEEE Trans. Automat. Control, vol. 63, no. 6, pp. 1798-1805, 2018.

[36] Z. Zhang, T. Leifeld, and P. Zhang, "Observer design for Boolean control networks," in Proc. 55th IEEE Conf. on Decision and Control (CDC2016), 2016, pp. 6272-6277.

[37] Y. Zhao, H. Qi, and D. Cheng, "Input-state incidence matrix of Boolean control networks and its applications," Systems Control Lett., vol. 59, no. 12, pp. 767-774, 2010. 